Pacific Journal of 


\title{
ON COVERINGS OF EUCLIDEAN SPACE BY CONVEX SETS
}

\author{
G. D. Chakerian and H. Groemer
}

Let $\mathscr{K}=\left\{K_{1}, K_{2}, \cdots\right\}$ be an infinite countable class of compact convex subsets of euclidean $n$-dimensional space $R^{n}$. We shall say that $\mathscr{K}$ permits a space covering or, more precisely, a covering of $R^{n}$, if there are rigid motions $\sigma_{1}, \sigma_{2}, \cdots$ such that $R^{n} \subset \bigcup_{i=1}^{\infty} \sigma_{i} K_{l}$. In this paper we concern ourselves with necessary and sufficient conditions in order that a given class $\mathscr{K}$ permits a space covering.

If the set of diameters $\left\{d\left(K_{i}\right): K_{i} \in K\right\}$ is bounded, the problem has already been solved in [3] by showing that in this case $\mathscr{K}$ permits a covering of $R^{n}$ if and only if the series $\sum_{i=1}^{\infty} v\left(K_{i}\right)$ of volumes $v\left(K_{\imath}\right)$ diverges. (The same result holds obviously without any restrictions on the diameters if $n=1$.) On the other hand, if $\left\{d\left(K_{i}\right)\right\}$ is unbounded and $n>1$, it is not difficult to see (cf. [1] and [2]) that the divergence of this series is no longer sufficient but only necessary. Only in the special case $n=2$ are some necessary and sufficient conditions known [2].

Our principal results are stated in the following $\$ 2$. Theorem 1 gives an inductive criterion that enables one to decide whether a given $\mathscr{K}$ permits a space covering. Theorems 2 and 3 serve the same purpose but are of a more explicit nature, involving the divergence of infinite series of geometric invariants associated with the members of $\mathscr{K}$. Other results, regarding coverings by balls, boxes (i.e. isometric images of $n$ dimensional intervals), and 2-dimensional sets, are stated and discussed in the same section. This is followed by the proofs of three lemmas in $\S 3$. Lemma 1 appears to be of some independent interest. $\$ 4$ contains the proofs of our theorems.

2. Theorems and corollaries. A nonempty compact convex set will be called a convex body. If $K$ is a convex body in $R^{n}$, and if $p, q$ are two points of $K$ such that the length of the segment $[p, q]$ is equal to the diameter $d(K)$, then we call the orthogonal projection of $K$ onto a hyperplane perpendicular to $[p, q]$ a normal projection of $K$. Of course, a normal projection of $K$ is not uniquely determined by $K$. However, if $\left\{K_{i}\right\}$ is given we shall always assume that for each $K_{\imath}$ a definite normal projection $N\left(K_{i}\right)$ has been selected and is kept fixed. Since each $N\left(K_{i}\right)$ is at most $(n-1)$-dimensional it is clear (using a self-explanatory extension of our original definition) what is meant by saying that the class 
$\left\{N\left(K_{i}\right)\right\}$ permits a covering of $R^{n-1}$. When a class $\left\{K_{i}\right\}$ is mentioned it is always assumed that $i=1,2, \cdots$.

THEOREM 1. Let $\mathscr{K}=\left\{K_{l}\right\}$ be a class of convex bodies $K_{i}$ in $R^{n}$ $(n \geqq 2)$ with diameters $d\left(K_{i}\right)$, volumes $v\left(K_{i}\right)$, and normal projections $N\left(K_{i}\right)$. Then, $\mathscr{K}$ permits a covering of $R^{n}$ if and only if either $\sum_{d\left(K_{1}\right) \leqq 1} v\left(K_{i}\right)=\infty$ or $\left\{N\left(K_{i}\right): d\left(K_{i}\right)>1\right\}$ permits a covering of $R^{n-1}$.

If $n \geqq 3$ it is clearly possible to apply the theorem again to the set $\left\{N\left(K_{i}\right): d\left(K_{t}\right)>1\right\}$, and so on. In this way one is lead to a criterion, expressing the property that $\mathscr{K}$ permits a space covering, entirely in terms of the convergence properties of infinite series. To formulate a result of this kind we introduce the following notations. For any convex body $K$ in $R^{n}(n \geqq 0)$ we set

$$
N^{0}(K)=K, \quad \text { and } \quad N^{m}(K)=N\left(N^{m-1}(K)\right) \quad(m=1,2, \cdots, n) .
$$

Furthermore, if $m=0,1, \cdots, n$ we write

$$
d^{m}(K)=d\left(N^{m}(K)\right) \quad \text { and } \quad v^{m}(K)=v\left(N^{m}(K)\right),
$$

where $v$ denotes the $(n-m)$-dimensional volume of $N^{m}(K)$, and define $v^{n}(K)=1, \quad d^{n}(K)=0 . \quad$ Obviously, $\quad d^{0}(K) \geqq d^{1}(K) \geqq \cdots \geqq d^{n}(K)=0$. Finally, if $n \geqq 1$, we partition the class $\left\{K_{i}\right\}$ of the preceding theorem into $n+1$ subclasses by the following definitions:

$$
\begin{aligned}
S^{0} & =\left\{K_{i}: d^{0}\left(K_{i}\right) \leqq 1\right\}, \\
S^{m} & =\left\{K_{i}: d^{m}\left(K_{i}\right) \leqq 1, d^{m-1}\left(K_{i}\right)>1\right\}(m=1,2, \cdots, n) .
\end{aligned}
$$

Using these definitions we can now state:

Theorem 2. A class $\left\{K_{i}\right\}$ of convex bodies $K_{i}$ in $R^{n}$ permits a covering of $R^{n}(n \geqq 1)$ if and only if one of the series $\Sigma_{K_{i} \in S^{m}} v^{m}\left(K_{i}\right)$ $(m=0,1,2, \cdots, n)$ diverges.

The condition in the preceding theorem can be expressed in terms of the divergence of a single series. This in turn can be expressed in terms of series involving the Minkowskian cross-sectional measures (Quermassintegrale) $W_{j}\left(K_{i}\right)$ of the convex bodies $K_{i}$, as defined, for example, in[5, p. 209].

THEOREM 3. Let $\left\{K_{i}\right\}$ be a given class of convex bodies in $R^{n}$ $(n \geqq 1)$, and let $d_{i}^{j}$ be defined by $d_{i}^{j}=d^{j}\left(K_{i}\right)$. Then, each of the following 
three conditions is necessary and sufficient that $\left\{K_{i}\right\}$ permits a covering of $R^{n}$.

$$
\begin{gathered}
\sum_{i=1}^{\infty} \frac{d_{i}^{0} d_{i}^{1} \cdots d_{l}^{n-1}}{1+d_{i}^{0}+d_{\imath}^{0} d_{i}^{1}+d_{i}^{0} d_{l}^{1} d_{\imath}^{2}+\cdots+d_{i}^{0} d_{i}^{1} d_{i}^{2} \cdots d_{i}^{n-2}}=\infty, \\
\sum_{i=1}^{\infty}\left(\frac{1}{v^{0}\left(K_{i}\right)}+\frac{1}{v^{1}\left(K_{i}\right)}+\cdots+\frac{1}{v^{n-1}\left(K_{\imath}\right)}\right)^{-1}=\infty, \\
\sum_{i=1}^{\infty} \frac{W_{0}\left(K_{i}\right)}{W_{1}\left(K_{i}\right)+W_{2}\left(K_{l}\right)+\cdots+W_{n}\left(K_{\imath}\right)}=\infty .
\end{gathered}
$$

To illustrate the applicability of these theorems consider first the case $n=2$. We have $S^{0}=\left\{K_{i}: d\left(K_{i}\right) \leqq 1\right\}, \quad S^{1}=\left\{K_{i}: d\left(K_{i}\right)>\right.$ $\left.1, d\left(N\left(K_{i}\right)\right) \leqq 1\right\}$, and $S^{2}=\left\{K_{i}: d\left(N\left(K_{i}\right)\right)>1\right\}$. Theorem 2 shows (combining $S^{1}$ and $S^{2}$ ) that $\left\{K_{i}\right\}$ permits a covering of $R^{2}$ if and only if either $\sum_{d\left(K_{i}\right) \leqslant 1} v\left(K_{i}\right)=\infty$ or $\sum_{d\left(K_{i}\right)>1} v\left(N\left(K_{i}\right)\right)=\infty$. The latter of these two conditions can also be written as $\Sigma_{d\left(K_{i}\right)>1} w\left(K_{i}\right)=\infty$, where $w\left(K_{i}\right)$ is the (minimum) width of $K_{l}$. This follows from the easily established fact that there are constants $c_{1}, \quad c_{2}$ such that $c_{1} v\left(N\left(K_{l}\right)\right) \leqq w\left(K_{i}\right) \leqq$ $c_{2} v\left(N\left(K_{i}\right)\right)$. These inequalities are, for example, a consequence of Lemma 1 . Thus, we have the following corollary, which expresses one of the main results of [2].

Corollary 1. Let $\mathscr{K}=\left\{K_{i}\right\}$ be a class of convex bodies in $R^{2}$, where $K_{i}$ has content $v\left(K_{i}\right)$ and (minimum) width $w\left(K_{i}\right)$. Then $\mathscr{K}$ permits a covering of $R^{2}$ if and only if either $\sum_{d\left(K_{i}\right) \leqq 1} v\left(K_{i}\right)=\infty$ or $\Sigma_{d\left(K_{i}\right)>1} w\left(K_{t}\right)=\infty$.

It should also be noted that the conditions given in [2] in order that $\left\{K_{i}\right\}$ permit a covering of $R^{2}$, involving the divergence of series constructed from the areas, perimeters, and diameters of the $K_{\imath}$, are immediate special cases of Theorem 3.

As another application we consider a class $\left\{B_{i}\right\}$ of closed balls $B_{i}$ in $R^{n}$. In this case all the sets $S^{m}$ are seen to be empty, except $S^{0}$ or $S^{n}$. If $d_{i}$ is the diameter of $B_{i}$ the two resulting series in Theorem 2 are (disregarding constant factors) $\Sigma_{d_{i} \leq 1} v\left(B_{i}\right)$ and $\Sigma_{d_{i}>1} 1$. The latter of these series diverges if and only if $\Sigma_{d_{i}>1} v\left(B_{i}\right)$ diverges (viz., if and only if there are infinitely many $B_{i}$ with $d_{i}>1$ ). Hence we find:

Corollary 2. Let $\mathscr{B}=\left\{B_{i}\right\}$ be a collection of closed balls $B_{i}$ in $R^{n}$. Then, $\mathscr{B}$ permits a covering of $R^{n}$ if and only if $\sum_{i=1}^{\infty} v\left(B_{i}\right)=\infty$.

This corollary shows also that the condition $\sum v\left(K_{i}\right)=\infty$, which is always necessary, and has been proved in [3] to be sufficient if the 
diameters are uniformly bounded, is also sufficient if the sets $K_{i}$ do not degenerate, in the sense that every $K_{i}$ has a circumscribed sphere and an inscribed sphere whose ratio of radii is bounded by a constant independent of $i$.

For the case of ellipsoids the following corollary is another immediate consequence of Theorem 2 .

COROllary 3. Let $\mathscr{E}=\left\{E_{i}\right\}$ be a class of $n$-dimensional ellipsoids, where $E_{i}$ has lengths of principal axes $e_{i}^{1} \geqq e_{i}^{2} \geqq \cdots \geqq e_{i}^{n}$. Then $\mathscr{E}$ permits a covering of $R^{n}$ if and only if one of the series

$$
\sum_{e_{i}^{1} \leqq 1} e_{i}^{1} e_{i}^{2} \cdots e_{i}^{n}, \quad \sum_{\substack{e_{1}^{1}>1 \\ e_{i}^{2} \leq 1}} e_{i}^{2} e_{i}^{3} \cdots e_{i}^{n}, \cdots, \sum_{\substack{e^{n-2}>1 \\ e_{i}^{n-1} \leqq 1}} e_{i}^{n-1} e_{i}^{n}, \quad \sum_{e_{i}^{n-1}>1} e_{i}^{n},
$$

diverges.

Finally we consider the case of boxes. If one wishes to apply Theorem 2 directly to this situation one meets the cumbersome problem of finding the normal projections (which are not projections in the directions of the edges). A more satisfactory solution is obtained by first inscribing in each box an ellipsoid of maximum volume and circumscribing an ellipsoid of minimal volume (considering first the case of a cube and then applying affine transformations). It is seen immediately that the boxes permit a covering if and only if the corresponding ellipsoids do. Furthermore, it is easily seen that the edges of the boxes and the corresponding lengths of the axes of the ellipsoids are proportional by a constant depending on $n$ only. Hence, we obtain from Corollary 3:

COROllary 4. Let $\mathscr{I}=\left\{I_{i}\right\}$ be a class of boxes, where $I_{i}$ has edge lengths $a_{i}^{1} \geqq a_{i}^{2} \geqq \cdots \geqq a_{i}^{n}$. Then $\mathscr{I}$ permits a covering of $R^{n}$ if and only if one of the series

$$
\sum_{a_{1} \leq 1} a_{i}^{1} a_{\imath}^{2} \cdots a_{i}^{n}, \quad \sum_{\substack{a_{1}^{1}>1 \\ a_{i} \leq 1}} a_{\imath}^{2} a_{i}^{3} \cdots a_{i}^{n}, \cdots, \sum_{\substack{a_{n}^{n-2}>1 \\ a_{1}^{n}-1 \leqq 1}} a_{i}^{n-1} a_{i}^{n}, \quad \sum_{a_{i}^{n-1}>1} a_{i}^{n},
$$

diverges.

For example, the class of boxes with edge lengths $a_{i}^{1}=i^{2}, a_{i}^{2}=1 / \sqrt{i}$, $a^{3}=1 / i$ does not permit a covering of $R^{3}$ although $\Sigma v\left(K_{i}\right)=\infty$, but the class with $a_{i}^{1}=\sqrt{ } i, a_{i}^{2}=\sqrt{i}, a_{i}^{3}=1 / i$ does.

\section{Three lemmas.}

LEMMA 1. Let $K$ be a convex body in $R^{n}$ that is contained in a right cylinder $Z$ of height $d(K)$ and base $N(K)$. Then $K$ contains a translate of $\frac{1}{3} Z$. 
Proof. There is no loss in generality by assuming that the points $a=(0,0, \cdots, 0, \alpha)(\alpha \geqq 0)$ and $-a$ are in $K$ and have the property that $2 \alpha=d(K)$. We may also assume that $N(K)$ lies in the plane $\left\{\left(x^{1}, x^{2}, \cdots, x^{n}\right): x^{n}=0\right\}$. The cylinder $Z$ can then be defined by $Z=$ $N(K)+[-a, a]$, and an arbitrary point $z \in Z$ can be represented in the form

$$
z=s a+p \quad(p \in N(K),-1 \leqq s \leqq 1) .
$$

Since the line through $z$ orthogonal to $N(K)$ contains a point of $K$, say $k$, we have

$$
k=r a+p \quad(-1 \leqq r \leqq 1)
$$

with the same $p$ as in (1). From (1) and (2) we obtain

$$
z=s a+p=s a+k-r a=k+\left(1+\frac{s-r}{2}\right) a+\left(1-\frac{s-r}{2}\right)(-a) .
$$

Because of $|s| \leqq 1,|r| \leqq 1$ we have $1+(s-r) / 2 \geqq 0,1-(s-r) / 2 \geqq$ 0 . Furthermore, we find $1+(1+(s-r) / 2)+(1-(s-r) / 2)=3$. Hence, the point $\frac{1}{3} z$ is a convex combination of the points $k, a,-a$ of $K$, and consequently $\frac{1}{3} z \in K$. Thus $\frac{1}{3} Z \subset K$ and the proof of the lemma is complete.

To formulate our second lemma we define a generalized $k$-cylinder as the vector sum $F+L$, where $F$ is a nonempty convex body of dimension not greater than $k(1 \leqq k \leqq n)$, and $L$ is an $(n-k)$ dimensional linear subspace that is orthogonal to $F$. The set $F$ will be called a base of the cylinder.

LEMMA 2. Let $C$ be a generalized $k$-cylinder $(1 \leqq k \leqq n)$ with base $F$ of $k$-dimensional volume $v_{k}(F)$, and let $B_{r}$ denote the ball in $R^{n}$ centered at the origin and of radius $r$. Then,

$$
v\left(C \cap B_{r}\right) \leqq \omega_{n-k} v_{k}(F) r^{n-k}
$$

where $\omega_{n-k}$ is the volume of the $(n-k)$-dimensional unit ball in $R^{n-k}$ $\left(\omega_{0}=1\right)$.

Proof. If the dimension of $F$ is less than $k$ then $v(C)=0$ and consequently $v\left(C \cap B_{r}\right)=0$. Since (3) is obviously satisfied in this case, we may now assume that $F$ has dimension equal to $k$. Then, for each point $x \in F$ there exists a unique $(n-k)$-dimensional affine subspace $L_{x}$ that contains $x$ and is orthogonal to $F$. Furthermore, 


$$
v\left(C \cap B_{r}\right)=\int_{x \in F} v_{n-k}\left(L_{x} \cap B_{r}\right) d x,
$$

where $v_{n-k}$ denotes the $(n-k)$-dimensional volume. Since $L_{x} \cap B_{r}$ is an $(n-k)$-dimensional sphere (or a single point, or empty) of radius at most $r$, we have $v_{n-k}\left(L_{x} \cap B_{r}\right) \leqq \omega_{n-k} r^{n-k}$, and (3) follows immediately from this inequality and (4).

For the formulation and the proof of our third lemma it is advantageous to introduce the following notation. Let $\phi$ and $\psi$ be two real valued functions on the class of all convex bodies of $R^{n}$. Then we write $\phi \approx \psi$ or $\phi(K) \approx \psi(K)$ if there are positive constants (depending on $\phi$ and $\psi$ only) such that $a \phi(K) \leqq \psi(K) \leqq b \phi(K)$ for all convex bodies $K$ of $R^{n}$.

LEMMA 3. Let $m$ be an integer with $0 \leqq m \leqq n$. Then,

$$
v^{m} \approx d^{m} d^{m+1} \cdots d^{n-1}
$$

and

$$
W_{m} \approx d^{0} d^{1} \cdots d^{n-m-1},
$$

where the right hand sides of these inequalities have to be set equal to 1 if $m=n$.

Proof. First we note that the case $m=n$ is completely trivial and can therefore be excluded from our further considerations. It is also clear that it suffices to prove (5) only in the case when $m=0$, since $v^{m}(K) \approx d^{m}(K) d^{m+1}(K) \cdots d^{n-1}(K)$ in $R^{n}$ expresses the same as $v^{0}\left(N^{m}(K)\right) \approx d^{0}\left(N^{m}(K)\right) \cdots d^{n-m-1}\left(N^{m}(K)\right)$ in $R^{m}$. If $n=1$ we have $v^{0}=d^{0}$ and $W_{0}=d^{0}$ which shows that both (5) and (6) are true in $R^{1}$. We make now the induction assumption that (5) (for $m=0$ ) and (6) be also true in spaces of dimensions less than $n$. Let $K$ be an arbitrary convex body of $R^{n}$. From Lemma 1 and the fact that $v(Z)=$ $d^{0}(K) v^{1}(K)$ we obtain

$$
v^{0} \approx d^{0} v^{1}
$$

(5) follows now from this relation and the fact that the induction assumption yields

$$
\begin{aligned}
v^{1}(K)=v^{0}(N(K)) \approx d^{0}(N(K)) d^{1}(N(K)) \cdots d^{n-2}(N(K)) \\
=d^{1}(K) d^{2}(K) \cdots d^{n-1}(K) .
\end{aligned}
$$

To prove (6) we denote again by $Z$ (or $Z(K)$ ) the cylinder associated with $K$ according to Lemma 1 . Then we have $Z=I+N$, where $I$ is a 
line segment of length $d^{0}(K)$ and $N=N(K)$. It has been shown (see [5], p. 215) that there are positive constants $a, b$ such that

$$
W_{m}(Z)=a d^{0}(K) W_{m}^{\prime}(N)+b W_{m-1}^{\prime}(N)
$$

where $W_{m}^{\prime}, W_{m-1}^{\prime}$ are cross-sectional measures with respect to $R^{n-1}$. The induction assumption implies

$$
\begin{aligned}
& W_{m}^{\prime}(N(K)) \approx d^{0}(N(K)) \cdots d^{n-m-2}(N(K))=d^{1}(K) \cdots d^{n-m-1}(K), \\
& W_{m-1}^{\prime}(N(K)) \approx d^{0}(N(K)) \cdots d^{n-m-1}(N(K))=d^{1}(K) \cdots d^{n-m}(K) .
\end{aligned}
$$

From these two relations and (7) we find (noting also that $d^{k+1}(K) \leqq$ $\left.d^{k}(K)\right)$

$$
W_{m}(Z(K)) \approx d^{0}(K) \cdots d^{n-m-1}(K)
$$

(6) is now an immediate consequence of (8) since Lemma 1 and the monotonicity, translation invariance and homogeneity of $W_{m}$ imply

$$
W_{m}(K) \approx W_{m}(Z(K))
$$

4. Proofs of the theorems. It is convenient to first prove Theorem 2 and then Theorem 1.

Proof of Theorem 2. First we wish to show that the condition

$$
\sum_{K_{i} \in S^{m}} v^{m}\left(K_{i}\right)=\infty, \text { for some } m=0,1, \cdots, n
$$

implies that $\left\{K_{i}\right\}$ permits a covering of $R^{n}$. If $n=1$ this is obvious, and we make the induction assumption that it be also true in $R^{k}$ if $k<n$. If $\Sigma_{K_{i} \in S^{0}} v^{0}\left(K_{i}\right)=\Sigma_{d\left(K_{i}\right) \leqslant 1} v\left(K_{i}\right)=\infty$ the desired conclusion has been shown to be true in [3]. For this reason we now assume that $n>1$ and that $\Sigma_{K_{i} \in S^{0}} v^{0}\left(K_{i}\right)<\infty$. Then, there is no loss in generality by assuming that actually $S^{0}=\varnothing$, i.e. $d\left(K_{\imath}\right)>1$ for all $K_{i}$. There is an integer $g$ such that $1 \leqq g \leqq n$ and $\Sigma_{K_{i} \in S^{8}} v^{g}\left(K_{i}\right)=\infty$. We now remark that for any divergent infinite series $\sum_{i=1}^{\infty} a_{i}$ with $a_{i} \geqq 0$ it is always possible to partition the indices into infinitely many disjoint subsets such that each of the corresponding subsequences $\left\{a_{1 \jmath}\right\} \quad\left\{a_{2 j}\right\} \cdots \quad$ satisfies $\sum_{j=1}^{\infty} a_{i j}=\infty$ $(i=1,2, \cdots)$ (First, let ụs define successively sums $s_{1}=a_{1}+a_{2}+\cdots+$ $a_{q_{1}}, s_{2}=a_{q_{1}+1}+\cdots+a_{q_{2}}, s_{3}=a_{q_{2}+1}+\cdots+a_{q_{3}}, \cdots$ so that $s_{r} \geqq 1$. The series $\sum_{h=0}^{\infty} s_{2 h+1}, \sum_{h=0}^{\infty} s_{2(2 h+1)}, \sum_{h=0}^{\infty} s_{4(2 h+1)}, \cdots$ are then divergent and determine obviously such a partitioning.) Applied to the above series this implies that $\left\{\boldsymbol{K}_{i}\right\}$ can be partitioned into pairwise disjoint subclasses $\left\{\boldsymbol{K}_{j}^{k}\right\}$ so that for $k=1,2, \cdots$ 


$$
\sum_{K_{j}^{k} \in S^{8}} v^{g}\left(K_{j}^{k}\right)=\infty .
$$

Since $g \geqq 1$ this can also be written as

$$
\sum_{N\left(K_{i}^{k}\right) \in S^{g-1}} v^{g-1}\left(N\left(K_{j}^{k}\right)\right)=\infty
$$

and according to the induction assumption it follows that $\left\{N\left(K_{j}^{k}\right)\right\}$ permits a covering of $R^{n-1}$ (for every fixed $k$ ).

To complete the proof we circumscribe about each $K_{j}^{k}$ a cylinder, say $Z\left(K_{j}^{k}\right)$, as indicated by Lemma 1 . Then, since $d\left(K_{j}^{k}\right) \geqq 1$ and thus the height of $Z\left(K_{j}^{k}\right)$ is not less than $1,\left\{Z\left(K_{j}^{k}\right)\right\}$ permits obviously a covering of a strip of width 1 (i.e. of the closed set between two parallel hyperplanes unit distance apart). The class $\left\{\frac{1}{3} Z\left(K_{j}^{k}\right)\right\}$ permits then a covering of a strip of width $1 / 3$. But since every $K_{j}^{k}$ contains a translate of $\frac{1}{3} Z\left(K_{j}^{k}\right)$ the class $\left\{K_{j}^{k}\right\}$ permits (for every fixed $k$ ) also a covering of a strip of width $1 / 3$. Since $R^{n}$ can be written as a union of strips of width $1 / 3$, the total class $\left\{\boldsymbol{K}_{i}\right\}=\left\{\boldsymbol{K}_{j}^{1}\right\} \cup\left\{\boldsymbol{K}_{j}^{2}\right\} \cup \cdots$ clearly permits a covering of $R^{n}$.

To prove the necessity of the conditions (9) we assume now that all the series $\sum_{K_{i} \in S^{m}} v^{m}\left(K_{i}\right)$ converge and $\left\{K_{i}\right\}$ covers $R^{n}$. The class $S^{n}$ consists then of only finitely many bodies $K_{i}$, and therefore $\Sigma_{K_{i} \in S^{n}} v\left(K_{i}\right) \leqq$ $c_{0}$ for some constant $c_{0}$. If $K_{i} \in S^{m}$, where $0 \leqq m<n$, let $Z_{i}=Z_{i}^{n-m}$ be a generalized $(n-m)$-cylinder with bases $N^{m}\left(K_{l}\right)$. Then $K_{i} \subset Z_{i}$, and if $\left\{K_{i}\right\}$ permits a covering of $R^{n}$ the class $\left\{Z_{i}\right\}$ must have the same property. In fact, we may assume that the cylinders $Z_{i}$ are already so arranged that

$$
R^{n} \subset \bigcup_{i=1}^{\infty} Z_{i}
$$

Now, let $r$ be a number that is greater than 1. According to Lemma 2

$$
v\left(B_{r} \cap Z_{\imath}^{n-m}\right) \leqq \omega_{m} v^{m}\left(K_{i}\right) r^{m}
$$

and therefore

$$
\begin{aligned}
v\left(B_{r} \cap \bigcup_{i=1}^{\infty} Z_{i}\right) \leqq c_{0}+\omega_{0} \sum_{K_{i} \in S^{0}} v^{0}\left(K_{i}\right)+\omega_{1} r \sum_{K_{i} \in S^{1}} & v^{1}\left(K_{i}\right)+\cdots \\
& +\omega_{n-1} r^{n-1} \sum_{K_{i} \in S^{n-1}} v^{n-1}\left(K_{i}\right) .
\end{aligned}
$$

Since all the series $\Sigma_{K_{i} \in S^{m}} v^{m}\left(K_{l}\right)$ are assumed to converge and $r>1$ there is a positive constant $c$ such that $v\left(B_{r} \cap \bigcup_{i=1}^{\infty} Z_{i}\right) \leqq c r^{n-1}$. However, (10) would imply $v\left(B_{r} \cap \bigcup_{i=1}^{\infty} Z_{i}\right)=\omega_{n} r^{n}$, giving a contradiction if $r$ is large enough. This completes the proof of Theorem 2. 
Proof of Theorem 1. If the assumptions of Theorem 1 are satisfied then either

$$
\sum_{d\left(K_{i}\right) \leqq 1} v\left(K_{i}\right)=\infty
$$

or one can apply Theorem 2 (for $R^{n-1}$ ) to the class $\left\{N\left(K_{i}\right): d\left(K_{i}\right)>\right.$ 1\}. This latter possibility leads immediately to the fact that

$$
\sum_{K_{1} \in S^{m}} v^{m}\left(K_{\imath}\right)=\infty, \text { for some } m=1,2, \cdots, n-1
$$

But if either (11) or (12) is satisfied, an application of Theorem 2 (for $R^{n}$ ) shows that $\left\{K_{l}\right\}$ permits a covering of $R^{n}$.

Conversely, if $\left\{K_{i}\right\}$ permits a covering of $R^{n}$, then the assumptions of Theorem 2 are satisfied, which means that either (11) holds or $\Sigma_{N\left(K_{i}\right) \in S^{m}} v^{m}\left(N\left(K_{t}\right)\right)=\infty$ for some $m=0,1, \cdots, n-1$. But, again by Theorem 2 (for $R^{n-1}$ ) the latter condition can be interpreted as stating that $\left\{N\left(K_{i}\right): d\left(K_{i}\right)>1\right\}$ permits a covering of $R^{n-1}$.

Proof of Theorem 3. First we concern ourselves with condition (a). It follows immediately from Lemma 3 that for $m=0,1, \cdots, n$ any of the series $\sum_{K_{i} \in s^{m}} v^{m}\left(K_{i}\right)$ which appear in Theorem 2 diverges if and only if the corresponding series $\Sigma_{K_{i} \in S^{m}} d^{m} d_{i}^{m+1} \cdots d_{i}^{n-1}$ diverges. If we write

$$
D_{i}=1+d_{\imath}^{0}+d_{i}^{0} d_{1}^{1}+\cdots+d_{i}^{0} d_{1}^{1} \cdots d_{\imath}^{n-2}
$$

we have

$$
\sum_{i=1}^{\infty} \frac{d_{i}^{0} \cdots d_{i}^{n-1}}{D_{i}}=\sum_{K_{i} \in S^{0}} \frac{d_{i}^{0} \cdots d_{i}^{n-1}}{D_{i}}+\sum_{K_{i} \in S^{1}} \frac{d_{i}^{0} \cdots d_{i}^{n-1}}{D_{i} \cdot}+\cdots+\sum_{K_{i} \in S^{n}} \frac{d_{i}^{0} \cdots d_{i}^{n-1}}{D_{i}}
$$

and we see that it suffices to prove that $\Sigma_{K_{i} \in s^{m}} d_{1}^{0} \cdots d_{i}^{n-1} / D_{i}$ diverges if and only if $\Sigma_{K_{i} \in S^{m}} d_{i}^{m} d_{i}^{m+1} \cdots d_{i}^{n-1}$ diverges. To show this equivalence we note that the definition of $S^{m}$ implies that for $K_{i} \in S^{m}$ and $m=0,1, \cdots, n$

$$
d_{\imath}^{n-1} \leqq \cdots \leqq d^{m} \leqq 1<d_{i}^{m-1} \leqq \cdots \leqq d_{i}^{0}
$$

where we have to omit for $m=0$ the terms $d_{i}^{m-1}, \cdots, d_{i}^{0}$ and for $m=n$ the terms $d_{i}^{n-1}, \cdots, d_{i}^{m}$. As a consequence of (13) we obtain for $m=$ $0,1,2, \cdots, n$

$$
d_{\imath}^{0} d_{i}^{1} \cdots d_{\imath}^{m-1} \leqq D_{i} \leqq n d_{\imath}^{0} d_{i}^{1} \cdots d_{\imath}^{m-1}
$$


(with $d_{i}^{0} d_{i}^{1} \cdots d_{i}^{m-1}=1$ if $m=0$ ). The equivalence of the conditions $\sum_{K_{i} \in S^{m}} d_{i}^{0} \cdots d_{i}^{n-1} / D_{\imath}=\infty$ and $\sum_{K_{i} \in S^{m}} d_{t_{t}}^{m} \cdots d_{i}^{n-1}=\infty$ is now an immediate consequence of (14).

Regarding the condition (b) of Theorem 3 we remark that because of Lemma 3

$$
\frac{1}{v^{m}(K)} \approx \frac{1}{d^{m}(K) \cdots d^{n-1}(K)}
$$

and that

$$
\frac{d_{i}^{0} \cdots d_{i}^{n-1}}{D_{i}}=\left(\frac{1}{d_{i}^{0} \cdots d_{i}^{n-1}}+\frac{1}{d_{i}^{1} \cdot \cdots d_{i}^{n-1}}+\cdots+\frac{1}{d_{i}^{n-1}}\right)^{-1} .
$$

These two relations show that (a) and (b) are equivalent.

To prove that (c) is necessary and sufficient we have only to point out that (a) and (c) are clearly equivalent because of (6).

It might be worth mentioning that the denominators in the series of condition (c) can be replaced by any other linear combination of the $W_{m}$ $(m=1,2, \cdots, n)$, provided that all coefficients of such a combination are positive. For example, if $B$ denotes the unit ball of $R^{n}$ it follows from Steiner's formula that $v(K+B)-v(K)$ can be used for this purpose. Hence, we have the following result:

$\left\{K_{i}\right\}$ permits a covering of $R^{n}$ if and only if

$$
\sum_{i=1}^{\infty} v\left(K_{i}\right) /\left(v\left(K_{i}+B\right)-v\left(K_{i}\right)\right)=\infty .
$$

\section{REFERENCES}

1. D. Chakerian, Covering space with convex bodies. The geometry of metric and linear spaces, Mich. 1974. Lecture Notes in Math. vol. 490, pp. 187-193. Springer-Verlag, Berlin-Heidelberg-New York 1975.

2. D. Chakerian and H. Groemer, On classes of convex sets that permit plane coverings, Israel J. Math., 19 (1974), 305-311.

3. H. Groemer, On a covering property of convex sets, Proc. Amer. Math. Soc., 59 (1976), 346-352.

4. - Some covering and packing problems, Amer. Math. Monthly, 83 (1976), 726-727.

5. H. Hadwiger, Vorlesungen über Inhalt, Oberfläche, und Isoperimetrie, Springer-Verlag, Berlin-Göttingen-Heidelberg 1957.

Received August 17, 1976. The second author was supported by National Science Foundation Research Grants MCS 76-06111 and MPS 71-03473.

UNIVERSITY OF CALIFORNIA

DAVIS, CA 95616

AND

UnIVERSITY OF ARIZONA

TUCSON, AZ 85721 


\section{PACIFIC JOURNAL OF MATHEMATICS EDITORS}

ICHARD ARENS (Managing Editor)

niversity of California

os Angeles, CA 90024

\section{. A. BEAUmont}

niversity of Washington

sattle, WA 98105

. C. MOORE

niversity of California

erkeley, CA 94720
J. DUGUNDJI

Department of Mathematics

University of Southern California

Los Angeles, CA 90007

R. FINN AND J. MiLgRAM

Stanford University

Stanford, CA 94305

\section{ASSOCIATE EDITORS}
F. BECKENBACH
B. H. NEUMANN
F. WOLF
K. YosHidA

\section{SUPPORTING INSTITUTIONS}

NIVERSITY OF BRITISH COLUMBIA

UNIVERSITY OF SOUTHERN CALIFORNIA

ALIFORNIA INSTITUTE OF TECHNOLOGY STANFORD UNIVERSITY

NIVERSITY OF CALIFORNIA

ONTANA STATE UNIVERSITY

UNIVERSITY OF HAWAII

NIVERSITY OF NEVADA

UNIVERSITY OF TOKYO

EW MEXICO STATE UNIVERSITY

UNIVERSITY OF UTAH

REGON STATE UNIVERSITY

NIVERSITY OF OREGON

WASHINGTON STATE UNIVERSITY

UNIVERSITY OF WASHINGTON

SAKA UNIVERSITY

AMERICAN MATHEMATICAL SOCIETY

The Supporting Institutions listed above contribute to the cost of publication of this Journal, but they a t owners or publishers and have no responsibility for its contents or policies.

Mathematical papers intended for publication in the Pacific Journal of Mathematics should be in typ Irm or offset-reproduced (not dittoed), double spaced with large margins. Underline Greek letters in re ierman in green, and script in blue. The first paragraph or two must be capable of being used separately as 'nopsis of the entire paper. Items of the bibliography should not be cited there unless absolutely necessary, hich case they must be identified by author and Journal, rather than by item number. Manuscripts, uplicate, may be sent to any one of the four editors. Please classify according to the scheme of Math. Revieu Idex to Vol. 39. All other communications should be addressed to the managing editor, or Elaine Bart Iniversity of California, Los Angeles, California, 90024.

100 reprints are provided free for each article, only if page charges have been substantially pai dditional copies may be obtained at cost in multiples of 50 .

The Pacific Journal of Mathematics is issued monthly as of January 1966. Regular subscription rate: \$72. year (6 Vols., 12 issues). Special rate: $\$ 36.00$ a year to individual members of supporting institutions.

Subscriptions, orders for numbers issued in the last three calendar years, and changes of address should ent to Pacific Journal of Mathematics, 103 Highland Boulevard, Berkeley, California, 94708.

UBLISHED BY PACIFIC JOURNAL OF MATHEMATICS, A NON-PROFIT CORPORATION

Printed at Jerusalem Academic Press, POB 2390, Jerusalem, Israel.

Copyright (C) 1978 Pacific Journal of Mathematics

All Rights Reserved 


\section{Pacific Journal of Mathematics}

\section{Vol. 75, No. $1 \quad$ September, 1978}

Mieczyslaw Altman, General solvability theorems

Denise Amar and Eric Amar, Sur les suites d'interpolation en plusieurs variables ..........................................

Herbert Stanley Bear, Jr. and Gerald Norman Hile, Algebras which satisfy a second order linear partial differential equation ..................

Marilyn Breen, Sets in $R^{d}$ having $(d-2)$-dimensional kernels ............

Gavin Brown and William Moran, Analytic discs in the maximal ideal space

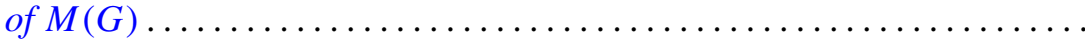

Ronald P. Brown, Quadratic forms with prescribed Stiefel-Whitney

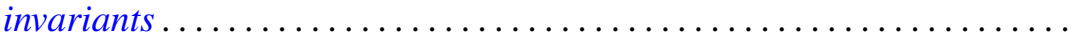

Gulbank D. Chakerian and H. Groemer, On coverings of Euclidean space by convex sets

S. Feigelstock and Z. Schlussel, Principal ideal and Noetherian groups.....

Ralph S. Freese and James Bryant Nation, Projective lattices ............

Harry Gingold, Uniqueness of linear boundary value problems for

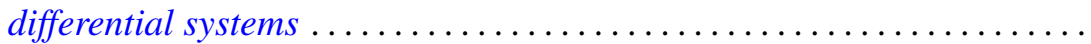

John R. Hedstrom and Evan Green Houston, Jr., Pseudo-valuation domains. . .

William Josephson, Coallocation between lattices with applications to measure extensions

M. Koskela, A characterization of non-negative matrix operators on $l^{p}$ to $l^{q}$ with $\infty>p \geq q>1$

Kurt Kreith and Charles Andrew Swanson, Conjugate points for nonlinear differential equations...........................

Shoji Kyuno, On prime gamma rings ........................ 185

Alois Andreas Lechicki, On bounded and subcontinuous multifunctions ..

Roberto Longo, A simple proof of the existence of modular automorphisms in approximately finite-dimensional von Neumann algebras ...

Kenneth Millett, Obstructions to pseudoisotopy implying isotopy for

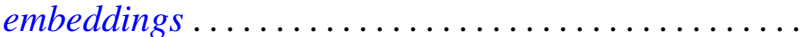

William F. Moss and John Piepenbrink, Positive solutions of elliptic equations. .

Mitsuru Nakai and Leo Sario, Duffin's function and Hadamard's

conjecture

Mohan S. Putcha, Word equations in some geometric semigroups ...

Walter Rudin, Peak-interpolation sets of class $C^{1} \ldots \ldots \ldots$

Elias Saab, On the Radon-Nikodým property in a class of locally convex

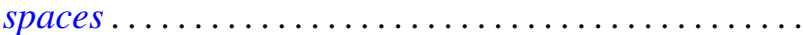

